\title{
Structure formation and characterization of EVAL membranes with cosolvent of isopropanol and water
}

\author{
Ruei-Liang Luo ${ }^{\mathrm{a}}$, Tai-Horng Young ${ }^{\mathrm{b}}$, Yi-Ming Sun ${ }^{\mathrm{a}, *}$ \\ ${ }^{a}$ Department of Chemical Engineering, Yuan Ze University, Chung-Li, Taiwan, ROC \\ ${ }^{\mathrm{b}}$ Institute of Biomedical Engineering, National Taiwan University, Taipei, Taiwan, ROC \\ Received 28 August 2002; accepted 4 October 2002
}

\begin{abstract}
Microporous poly (ethylene-co-vinyl alcohol) (EVAL) membranes can be prepared by the solution casting method in combination of thermally induced phase separation (TIPS) with solvent evaporation. A cosolvent of isopropanol and water (3:2 w/w) is used to prepare the casting solution. EVAL membranes of different bulk or true densities, porosity, flexibility, crystallinity, and structure can be obtained by adjusting the casting temperature. A pseudo-binary temperature-concentration phase diagram of the EVAL-cosolvent system has been proposed, based on the information obtained from SEM, DSC, and light transmittance studies, to account for the membrane formation mechanism. The dense membranes were obtained when the casting temperature was higher than the upper critical solution temperature (UCST) of the system, but they might contain some nonSEM-observable micropores if the casting temperature was below the glass transition temperature $\left(T_{\mathrm{g}}\right)$ of EVAL. The porous and the least crystalline membranes with a honeycomb-like morphology were obtained through liquid-liquid demixing and vitrification at a casting temperature between the UCST and the dynamic crystallization temperature $\left(T_{\mathrm{c}}\right)$. Highly porous and crystalline membranes were obtained when they were prepared at temperatures near $T_{\mathrm{c}}$. In this case, the particulate membranes were resulted from solid-liquid demixing mainly via TIPS, and membranes with leafy morphology were created through liquid-liquid demixing and then followed with immediately crystallization.
\end{abstract}

(C) 2002 Elsevier Science Ltd. All rights reserved.

Keywords: Membrane formation; Phase separation; Phase diagram

\section{Introduction}

Phase inversion technique is a common method to tailor the structure of the membrane for particular applications [1-3]. Typical examples are the microporous membranes used in microfiltration and ultrafiltration processes and the asymmetric membranes used in reverse osmosis and gas or pervaporation separations [3]. Phase separation during the membrane formation process creates the porous structure within the membranes. Immersion of a casting polymer solution into a nonsolvent bath is a common way to induce the phase inversion, and it is called as nonsolvent induced phase separation, or immersion precipitation method [4-7]. In this process, phase separation due to spinodal decomposition or nucleation and growth occurs, gelation and solidification of the polymer-rich phase are followed, and then porous

\footnotetext{
* Corresponding author. Tel.: +886-3-4638800x558; fax: 886-34559373.

E-mail address: cesunym@saturn.yzu.edu.tw (Y.M. Sun).
}

structure within the membrane can be formed. The polymer precipitation is best represented by the movement of a line of pathway through a solvent-nonsolventpolymer ternary phase diagram [7]. By varying the preparation conditions, a lot of desired membrane structures could be produced [2,3]. Moreover, if the polymer casting solution has an UCST or the polymer can be crystallized from the homogeneous solution, phase separation can also be induced by reducing the casting temperature to a temperature lower than the UCST or crystallization temperature. If the polymer solidifies or crystallizes before it collapses together, a porous membrane is formed after the solvent is removed. This process is known as thermal-inversion [8], thermally induced phase separation (TIPS) [9-12], thermal precipitation [2], or polymer precipitation by cooling [3], and TIPS is the most acceptable one and is used in this article.

Recently, Young and his coworkers have published a series of papers on the immersion precipitation technique for the preparation of porous poly(ethylene-co-vinyl 
alcohol) (EVAL) membranes. Solvent-nonsolvent-polymer systems of DMSO-water-EVAL [13-18] or DMSOisopropanol-EVAL [18] have been extensively studied. In addition, the use of two nonsolvents (isopropanol-water) as a cosolvent for the EVAL membranes preparation was investigated $[19,20]$. A homogeneous solution, liquidliquid or solid-liquid two-phase mixture can exist at $60{ }^{\circ} \mathrm{C}$. A ternary phase diagram of isopropanol-water-EVAL at $60{ }^{\circ} \mathrm{C}$ has been provided to explain the formation mechanism of porous EVAL membranes [20]. In addition, solid EVAL polymer can precipitate from a homogeneous solution prepared at $60{ }^{\circ} \mathrm{C}$ if the temperature is reduced to the room temperature. It is suggested that it is also possible to use TIPS technique to prepare porous EVAL membranes. However, the application of TIPS technique has never been discussed for the preparation of porous EVAL membranes to the best knowledge of the authors.

In this work, the application of the above mentioned cosolvent of isopropanaol and water for the preparation of EVAL membranes at various temperatures is reported. Neither isopropanol nor water can dissolve EVAL individually, but a certain mixture of isopropanol and water become a solvent for EVAL. A clear and homogeneous solution can be formed by dissolving $15 \%$ EVAL polymer in a cosolvent of isopropanol and water $(3: 2 \mathrm{w} / \mathrm{w})$ at $60{ }^{\circ} \mathrm{C}$. Solid polymer could be obtained either by reducing the temperature or by evaporating the cosolvent. The polymer solution was cast on a glass plate pre-equilibrated at a certain temperature. Depending on the membrane casting temperature, the structure morphology, densities, and crystallinity of the membranes may be significantly different. Apparently, the membrane formation process is not a simple TIPS as commonly recognized in the literature, in which a solvent with high boiling point is used [2,3, $8-12]$. The polymer precipitation by solvent evaporation is also important in our case because the cosolvent is quite volatile. In our study, light transmittance experiments were carried out for both closed and open, i.e. solvent evaporation inhibited and allowed, systems to identify the membrane formation mechanism. Differential scanning calorimetry (DSC) was used to determine the dynamic crystallization temperature $\left(T_{\mathrm{c}}\right)$ of the polymer prepared from the casting solution and the crystallinity of the prepared membranes. Since we cannot differentiate the evaporation of isopropanol or water, the polymer solution is treated as a binary polymer-cosolvent system. The phase behaviors during the membrane formation process are discussed and a plausible phase diagram is proposed to facilitate the explanation of the membrane formation mechanism. Although the discussion is based on a pseudo-binary assumption, it leads us into a preliminary understanding of the system and gives clues for future studies. The correlation between the permeation properties for some selected drugs through those membranes and the membrane structure will be presented elsewhere.

\section{Experimental}

\subsection{Materials}

Poly(ethylene-co-vinyl alcohol) (EVAL) with ethylene content of $44 \mathrm{~mol} \%$ and $\overline{M_{\mathrm{v}}}=56,000$ was obtained from Kuraray Co. Ltd, Japan (E105A). Isopropanol of reagent grade was purchased from Merck (USA). Deionized water prepared by Millipore (USA) QSP system was used in this study.

\subsection{Membrane preparation}

The membranes were prepared by the solution casting method. EVAL polymer (15 wt $\%)$ was dissolved in the cosolvent of isopropanol and water $\left(3: 2\right.$ by weight) at $60{ }^{\circ} \mathrm{C}$. The polymer solution was poured on a glass plate which had been equilibrated in a air-circulated oven at temperatures of $5,20,30,45$, and $60{ }^{\circ} \mathrm{C}$. The thickness of the casting solution was controlled at $200 \mu \mathrm{m}$ with a glass casting knife. The cast membranes were kept in the oven at the casting temperature to evaporate the solvent for $24 \mathrm{~h}$. The membranes were removed from the glass plate by immersing them in water at the room temperature, and then dried in vacuum for $24 \mathrm{~h}$. These membranes were named as EVAL-5, EVAL-20, EVAL-30, EVAL-45, and EVAL-60 according to the membrane preparation temperature used (5, $20,30,45$, and $60{ }^{\circ} \mathrm{C}$, respectively). The averaged membrane thickness was measured at 10 different points in the membrane with a digital thickness gauge (Mitutoyo, IDF-112). The resulting membranes were about 20-50 $\mu \mathrm{m}$ in thickness depending on the preparation temperature.

\subsection{Solution behaviors}

The casting solution (15 wt\% EVAL polymer in the cosolvent) was completely transparent at the preparation temperature $\left(60^{\circ} \mathrm{C}\right)$, indicated completely mixing. However, it became turbid gradually with the time, as a sign of phase separation, if the casting temperature was lower than a certain value. The change of the light transmittance along with time was determined for the casting solution at various temperatures. The casting solution (prepared at $60{ }^{\circ} \mathrm{C}$ ) was poured into a standard UV/Vis cuvette with light path of $1 \mathrm{~cm}$. The solution was quenched to a selected temperature thermostated by a water jacket, which was built in the cuvette holder. The light transmittance of the solution at wavelength of $314 \mathrm{~nm}$ was monitored by a Hewlett Packard 8452 A UV/Vis spectrophotometer. Since the light path through the sample is $1 \mathrm{~cm}$, the transmittance can go to absolute zero if there is a complete phase separation in the polymer solution. It should be noted that the light transmittance of the solution was followed with the time without any evaporation of solvent. The experiments were treated as a closed system operation. The phase separation is only due to the temperature change in the solution. These 
precipitates of the solid polymer samples formed at lower temperatures $\left(5,15\right.$, and $\left.30^{\circ} \mathrm{C}\right)$ in this experiment were collected for further analysis in order to make comparison with the membranes prepared by the procedures described above. These samples were named as EVAL-5C, EVAL$15 \mathrm{C}$, and EVAL-30C according to the corresponding sample preparation temperature $\left(5,15\right.$, and $30^{\circ} \mathrm{C}$, respectively) used.

An actual membrane preparation process involves solvent evaporation. Phase separation occurs due to the combination of temperature change and solvent evaporation. The phenomenon was observed by using an open light transmittance device [21]. The device included a $\mathrm{He}-\mathrm{Ne}$ laser light source (Uniqphase), a photo sensor and digital storage oscilloscope (Hitachi, VC-6025), and a hot (also cool) stage (Linkam, THMS 30). The casting solution (prepared at $60{ }^{\circ} \mathrm{C}$ ) was poured on a glass plate, and then placed on the hot stage equilibrated at a selected temperature. The experiment was operated similar to the actual membrane preparation process and was considered as an open system operation. Both the temperature change and solvent evaporation can induce the phase separation in the casting solution. The light transmittance of the samples was recorded with the time. Depending on the casting temperature, the membranes prepared can be completely transparent or opaque. The reported data were normalized in the range between the transmittance of the initial casting solution and the lowest transmittance obtained in the experiments.

\subsection{Scanning electron microscopy (SEM)}

The structure of membranes or solid polymer samples formed in a closed system was examined by a scanning electron microscope (Jeol, JSM-5600). The membranes were sectioned under liquid nitrogen. The solid polymer samples obtained from the closed light transmittance experiments were fractured directly at the room temperature. Each sample was coated with gold by ion spurting under vacuum before SEM observation.

\subsection{Density measurement}

The true density $\left(\rho_{\mathrm{t}}\right)$ of a membrane and a solid polymer sample was determined by using a pycnometer (Micromeritics 1305) to determine the exact sample volume. Helium gas was used in the measurement and the true volume of a sample (solid portion of the sample where helium is unable to enter) was determined, and then the true density could be calculated if the weight of the sample was known. The true volume is considered as the volume of the solid polymer body which contains no 'pore' bigger than helium molecule.

The bulk density $\left(\rho_{\mathrm{b}}\right)$ of a sample was determined based on the exterior dimension of the sample. The bulk volume, which includes the solid polymer portion and the porous portion of the sample, was determined, and the bulk density was calculated when the sample weight was available.

The porosity $(\epsilon)$ of the sample could be estimated according to $\epsilon=1-\rho_{\mathrm{b}} / \rho_{\mathrm{t}}$.

\subsection{Thermal analysis}

The thermal behaviors at the melting points of the membranes and the solid polymer samples obtained from the closed light transmittance experiments were evaluated by a Perkin-Elmer DSC-7 in the temperature range from 100 to $200{ }^{\circ} \mathrm{C}$ with a raising rate of $10^{\circ} \mathrm{C} / \mathrm{min}$. The membranes or samples were grinded and dried before the experiment. A sample of 5-7 mg in weight was sealed in an aluminium pan $(10 \mu \mathrm{l})$ for each test.

When the temperature of the polymer solution is reduced, EVAL solid (crystals) can precipitate from the solution at a certain temperature. The crystallization temperature depends on the temperature reduction rate and it is called as dynamic crystallization temperature $\left(T_{\mathrm{c}}\right)$. DSC can be used to determine $T_{\mathrm{c}}$. The casting solution (16-19 $\mathrm{mg}$ ) was sealed in an aluminium liquid sample pan $(50 \mu \mathrm{l})$. In each run, the temperature was kept at $60{ }^{\circ} \mathrm{C}$ for $20 \mathrm{~min}$, and then cooled at various rates $\left(20,15,10,5,1\right.$, and $\left.0.5^{\circ} \mathrm{C} / \mathrm{min}\right)$ to $-40{ }^{\circ} \mathrm{C}$. A heat-releasing peak was observed at each run. The onset of the peak was used to determine $T_{\mathrm{c}}$. Because the sample was liquid solution at $60{ }^{\circ} \mathrm{C}$, leakage-free condition had to be checked before each run. It was confirmed that no detectable weight loss was identified after the sealed sample had been equilibrated at $70{ }^{\circ} \mathrm{C}$ for at least $2 \mathrm{~h}$.

\section{Results and discussions}

\subsection{Membrane characterization}

The membranes formed at different casting temperatures showed significant differences in external appearance and internal structure. The membranes formed at 60 and $45^{\circ} \mathrm{C}$ (EVAL-60 and EVAL-45, respectively) were colorless and transparent, however, those formed at $30^{\circ} \mathrm{C}$ and below (EVAL-30, EVAL-20, and EVAL-5) were opaque. The typical scanning electron micrographs of the cross-section of the membranes of two magnifications are shown in Fig. 1. Apparently dense structure was obtained for membranes EVAL-60 and EVAL-45, and there was not much difference between the pictures obtained from both membranes so that only the pictures for EVAL-45 were shown (Fig. 1(a)). Porous structure was obtained for membranes formed at $30{ }^{\circ} \mathrm{C}$ and below. Their internal structure was quite different for EVAL-30, EVAL-20, and EVAL-5 (Fig. 1(b)-(d), respectively); however, all the porous membranes contained spherical particles (nodules) within their interior texture and the particle size decreased as the membrane preparation temperature lowered. The spherical particles were enclosed in a honeycomb-like structure in EVAL-30 membranes, and 
(a)
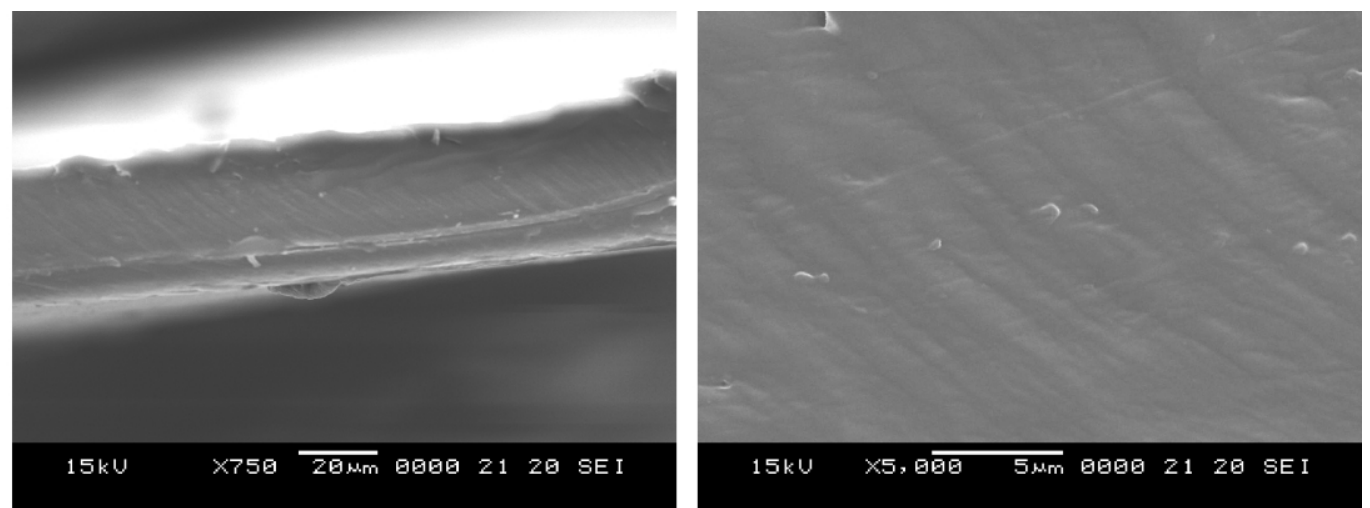

(b)
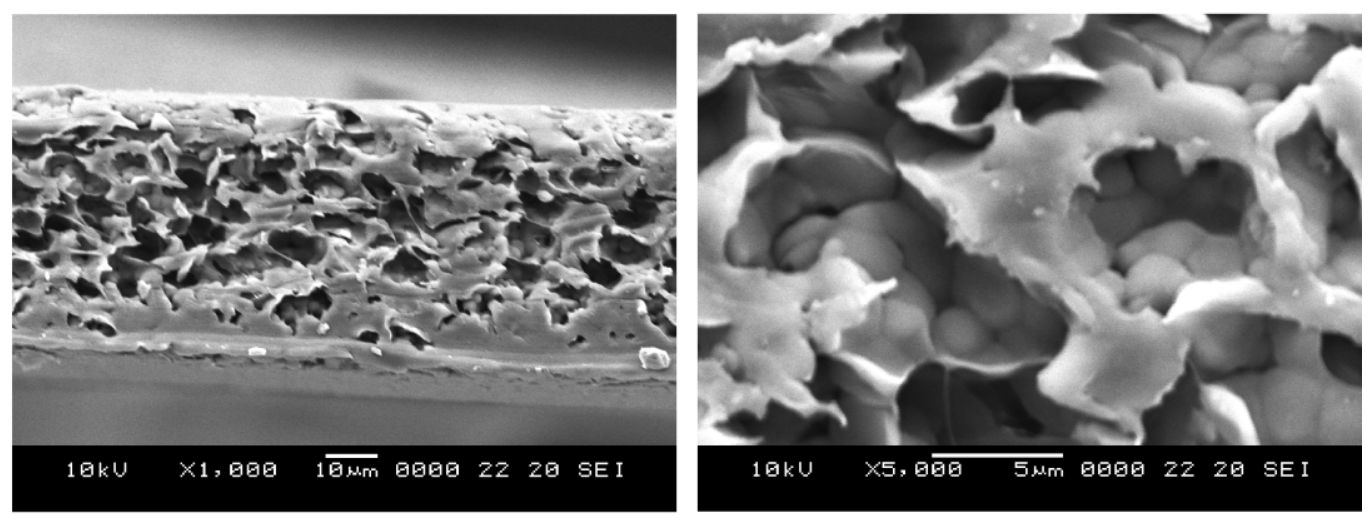

(c)
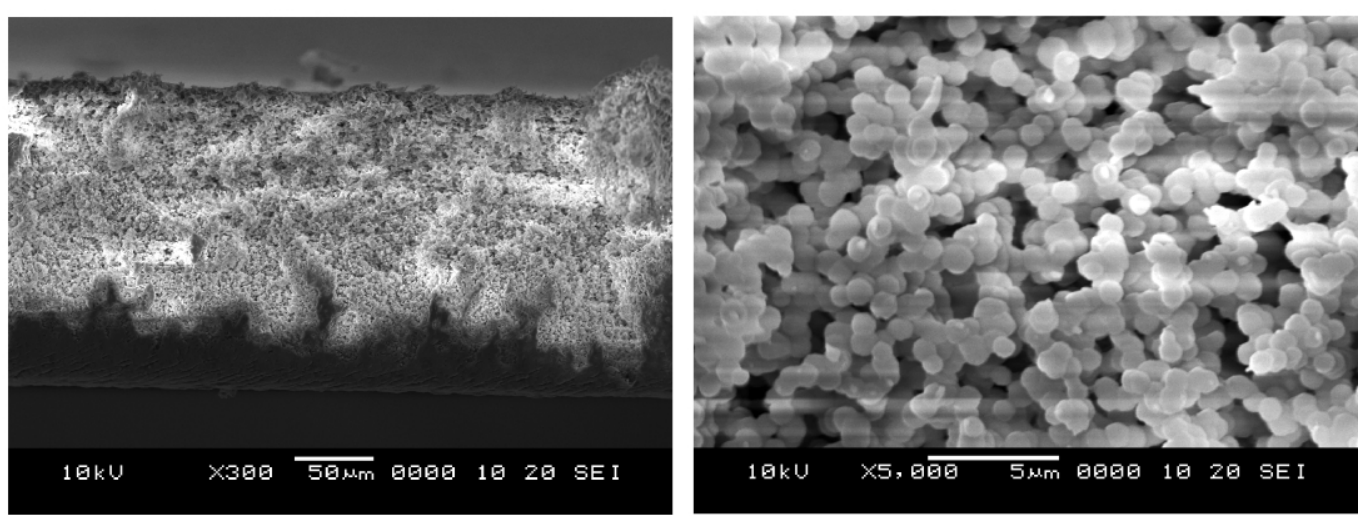

(d)
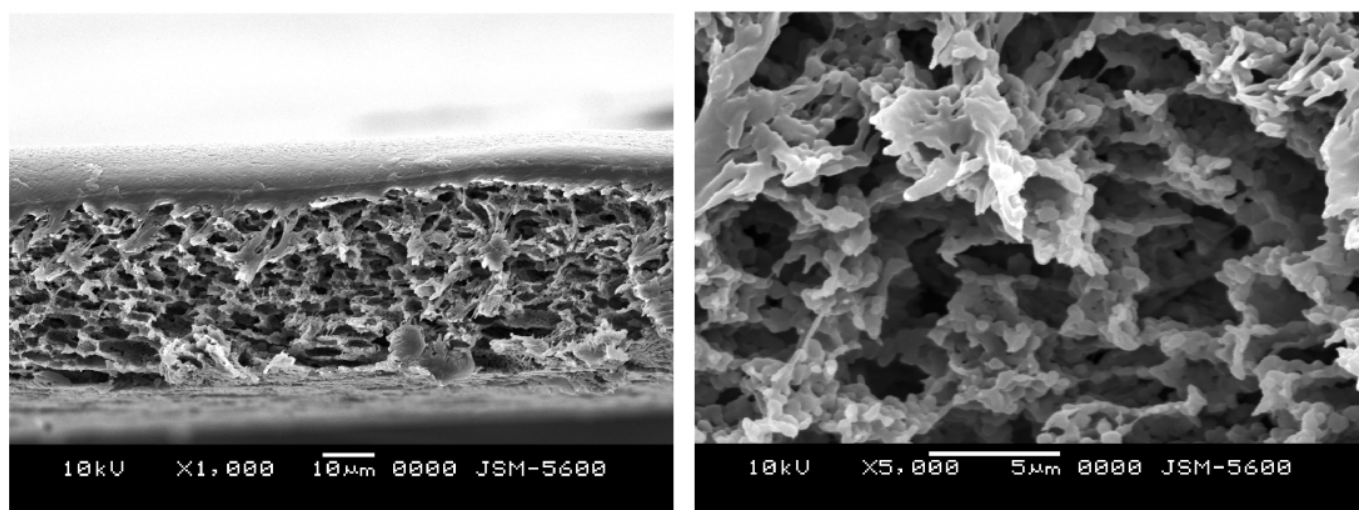

Fig. 1. SEM pictures of the cross-section of the membranes prepared at various temperatures. (a) EVAL-45, (b) EVAL-30, (c) EVAL-20, and (d) EVAL-5. 


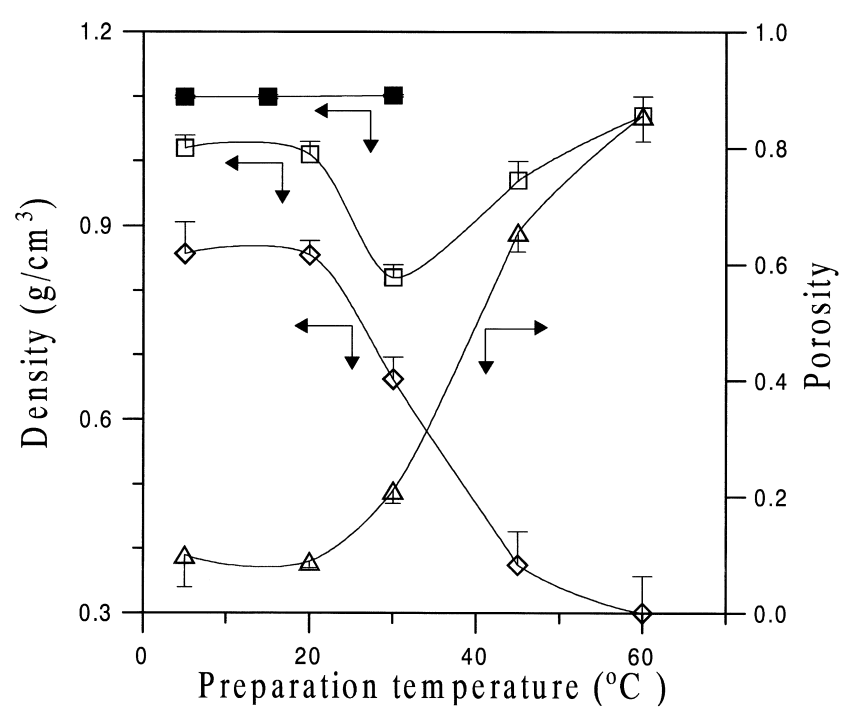

Fig. 2. The bulk density ( $\triangle$ : left axis), true density ( $\square$ : left axis), and porosity $(\diamond$, right axis) for the membranes and the true density of solid polymer samples ( $\boldsymbol{\square}$ : left axis) prepared at various temperatures.

each cell of the honeycomb structure formed a closed pore. The particles in the EVAL-20 membranes were connected together, the space between those unconnected portions formed open pores, and resulted in a foam-like structure overall. The particles in the EVAL-5 membranes were highly connected to become flake-like or leafy structure, and the space in-between formed open pores.

It was found that the bulk density, the true density, and the porosity of the membranes depended on the membrane preparation temperature. The density and the porosity of the membranes were shown in Fig. 2. The bulk density and true density were almost the same for the membranes formed at $60{ }^{\circ} \mathrm{C}$ (EVAL-60) and the porosity of the membrane was negligible. EVAL-60 could be considered as a 'dense' membrane. The membrane density $\left(1.07 \pm 0.03 \mathrm{~g} / \mathrm{cm}^{3}\right)$ was slightly lower than the reported manufacturer datum $\left(1.14 \mathrm{~g} / \mathrm{cm}^{3}\right)$ and our measured datum $\left(1.12 \mathrm{~g} / \mathrm{cm}^{3}\right)$ for the EVAL pellets. The difference might be due to the lower packing density for materials by the casting process than that by the melt extrusion.

Apart from the one formed at $60{ }^{\circ} \mathrm{C}$, the true density and the bulk density are different for all the other membranes formed at $45^{\circ} \mathrm{C}$ and below and the porosities of those membranes could be determined. Although the membranes formed at $45^{\circ} \mathrm{C}$ (EVAL-45) did not have any SEMobservable-pores (at magnification of $15,000 \times$ ), its porosity was estimated to be about 0.08 . It may contain micropores (nano-scale pores) for the accommodation of helium molecules but not observable by SEM. For the membranes formed at $30{ }^{\circ} \mathrm{C}$ (EVAL-30), the bulk and true densities were much smaller than those for EVAL-45 and EVAL-60, and the porosity of EVAL-30 became about 0.4 . The porous structure can be clearly observed in the SEM pictures (Fig. 1(b)). For both the membranes formed at 20 and $5{ }^{\circ} \mathrm{C}$ (EVAL-20 and EVAL-5, respectively), they had

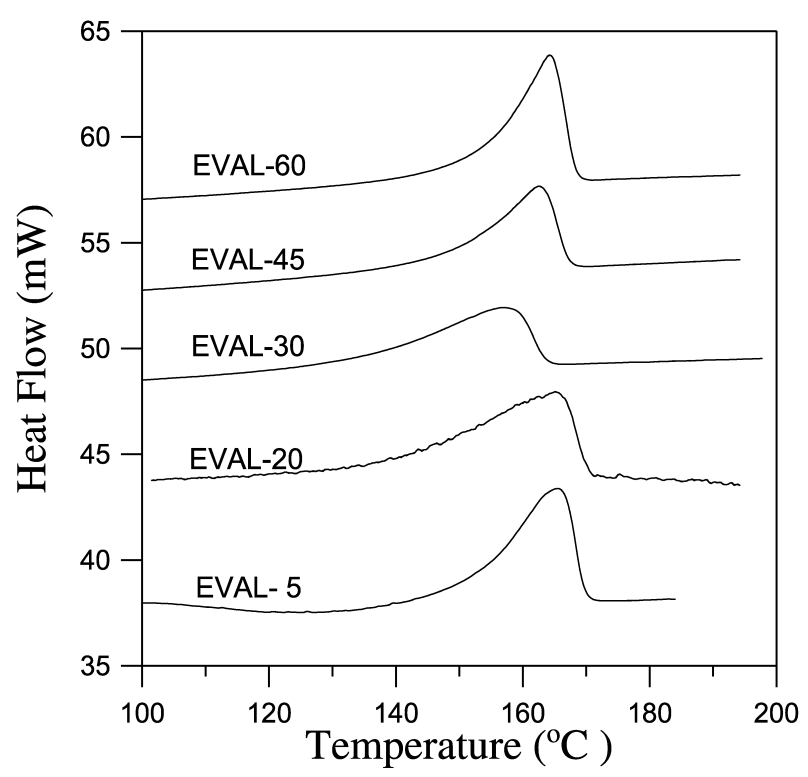

Fig. 3. DSC diagrams of the melting curves of the EVAL membranes prepared at various temperatures. (Scanning rate: $10^{\circ} \mathrm{C} / \mathrm{min}$ ).

nearly the same values of densities and porosity although their inner structures were quite different. Their true densities were larger than those of EVAL-45 and EVAL30 , but were slightly smaller than that of EVAL-60, and their bulk density went down further compared to that of EVAL-30. Membranes EVAL-20 and EVAL-5 had the highest porosity (0.6) among all the membranes. In fact, EVAL-20 and EVAL-5 were quite fragile for any direct application.

Due to the structural difference, the membrane formation temperature also affects the crystallinity of the membranes. The melting behaviors of these membranes were shown in Fig. 3, and the corresponding melting heats and the peak temperatures of those membranes were summarized in Table 1. The trend of the results was similar to the true densities of the membranes as both the melting heat and peak temperature decreased first and then increased to a relative stable value when the preparation temperature was decreased from 60 to $5^{\circ} \mathrm{C}$. It is well known that the melting heat is a direct measure of the crystallinity of a polymer. The membrane prepared at $30{ }^{\circ} \mathrm{C}(\mathrm{EVAL}-30)$ showed the lowest

Table 1

Melting heat and peak temperature for the membranes or solid polymer samples prepared at various temperatures

\begin{tabular}{lll}
\hline Sample & $\Delta H_{\mathrm{m}}(\mathrm{J} / \mathrm{g})$ & Peak temperature $\left({ }^{\circ} \mathrm{C}\right)$ \\
\hline EVAL-60 & $60.5 \pm 0.4$ & $164.2 \pm 0.2$ \\
EVAL-45 & $58.6 \pm 0.4$ & $162.6 \pm 0.2$ \\
EVAL-30 & $51.5 \pm 0.5$ & $157.1 \pm 0.3$ \\
EAVL-20 & $67.6 \pm 0.8$ & $165.1 \pm 0.6$ \\
EVAL-5 & $68.8 \pm 0.5$ & $165.7 \pm 0.3$ \\
EVAL-30C & $72.3 \pm 0.7$ & $166.2 \pm 0.4$ \\
EVAL-15C & $71.8 \pm 0.8$ & $166.1 \pm 0.4$ \\
EVAL-5C & $72.1 \pm 0.8$ & $166.2 \pm 0.3$ \\
\hline
\end{tabular}


crystallinity among all the membranes. When the membrane preparation temperature was above $30^{\circ} \mathrm{C}$, the crystallinity increased with the preparation temperature. The crystallinities for the membranes obtained at $20-5^{\circ} \mathrm{C}$ (EVAL-20 and EVAL-5, respectively) were almost the same and were the highest value among all the membranes.

In a summary, the membranes obtained at various temperatures can be described as follows: (1) EVAL-60: transparent, dense, moderate crystalline, and flexible film; (2) EVAL-45: transparent, microporous but not observable by SEM, moderate crystalline, and flexible film; (3) EVAL30: opaque, honeycomb-like and moderate porous, least crystalline, and flexible film; (4) EVAL-20 and EVAL-5: both are opaque, highly porous, highly crystalline, and fragile films, but the structure of EVAL-20 is foam-like and that of EVAL-5 is flake-like (leafy).

It is evident that the porous membranes obtained in this study have experienced a composite process of solution phase separation, solvent evaporation, and polymer solidification or crystallization, which happened simultaneously or consequently. The difference in membrane structure and crystallinity was highly related to the composite process. In order to understand better the membrane formation mechanism, several experiments have been carried out to characterize those phenomena. Light transmittance study was used to identify the occurrence of phase separation; DSC was used to obtain the crystallization temperature of the polymer in the solution. The studies operated in a closed system could isolate the phase separation due to temperature change from the effect of solvent evaporation.

\subsection{Phase behaviors of the polymer casting solutions}

It is generally true that the light transmittance through a polymer solution drops when there occurs a phase

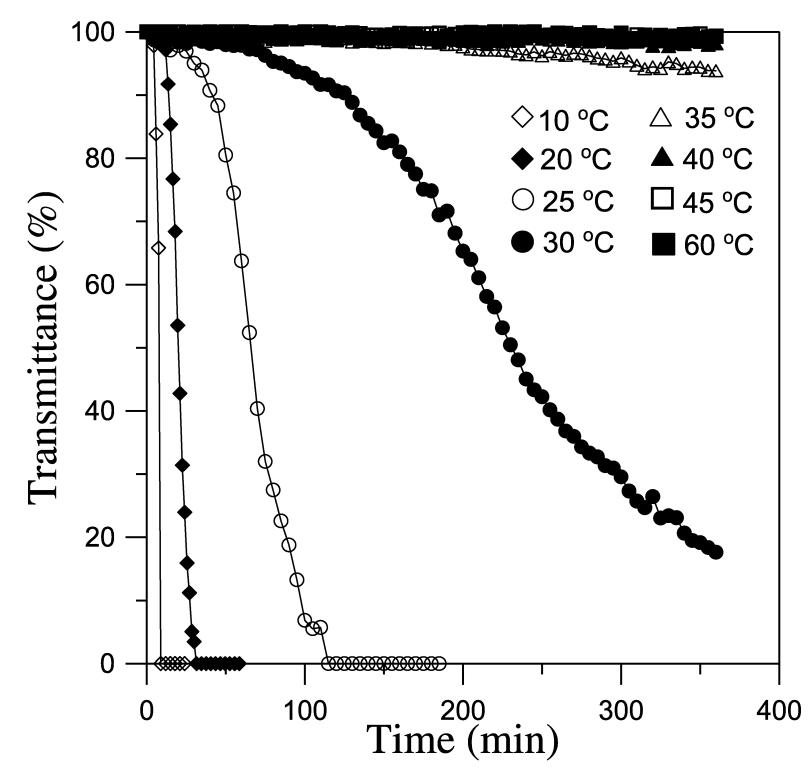

Fig. 4. Light transmittance-time curves for EVAL solutions in a closed system at various temperatures. (a)

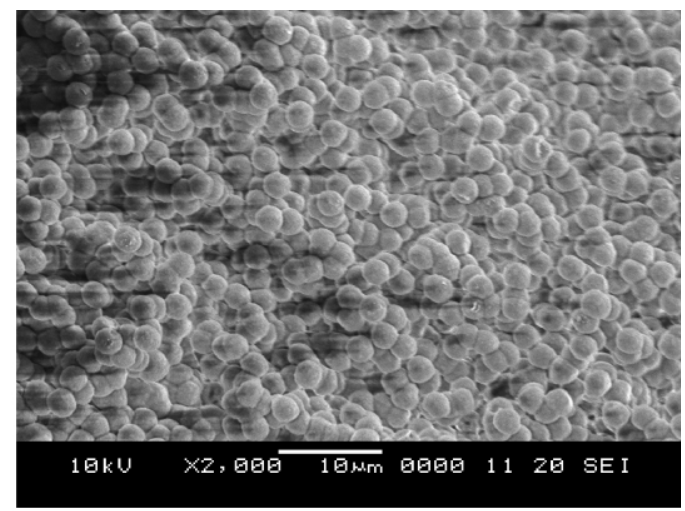

(b)

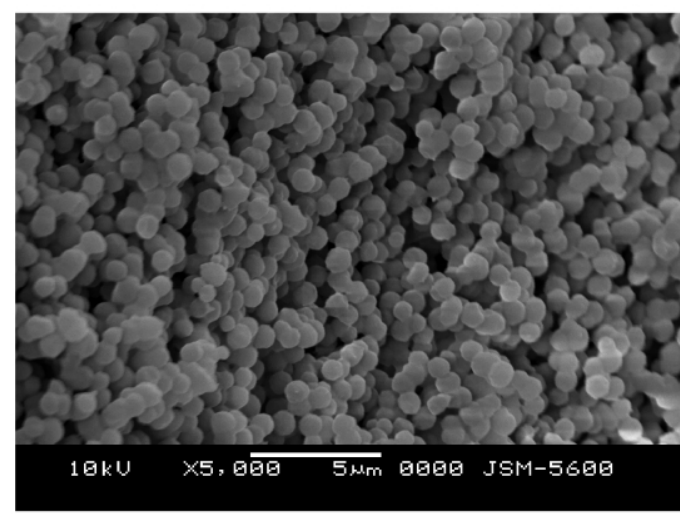

(c)

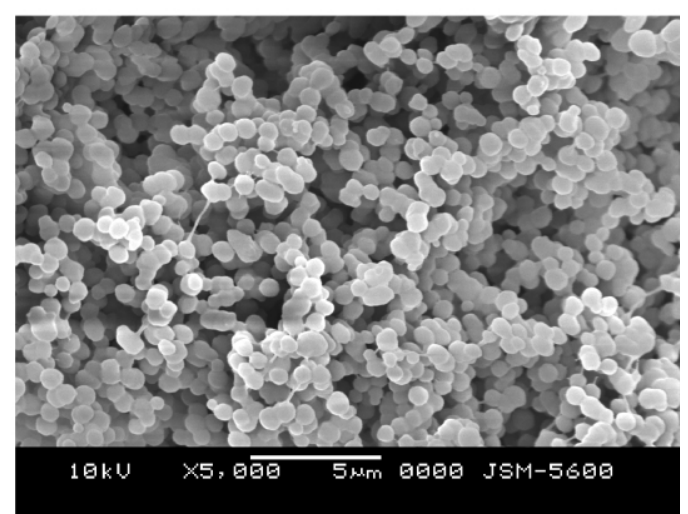

Fig. 5. SEM photographs for solid EVAL samples prepared in a closed system at various temperatures. (a) EVAL-30C, (b) EVAL-15C, and (c) EVAL-5C.

separation or a transition from a homogeneous phase to a heterogeneous phase due to the difference of the refractive indices of different phases. Light transmittance experiments were carried out in this study to identify the solution phase behaviors upon membrane preparation. In the process of membrane formation under study, the casting solution may experience the temperature change as well as the concentration change due to solvent evaporation. Both of the factors could lead to phase separation due to a change in polymer solution thermodynamics. In order to differentiate the influences from both factors, a light transmittance study with a closed system was carried out to monitor the phase behavior upon change in the solution temperature only, and 


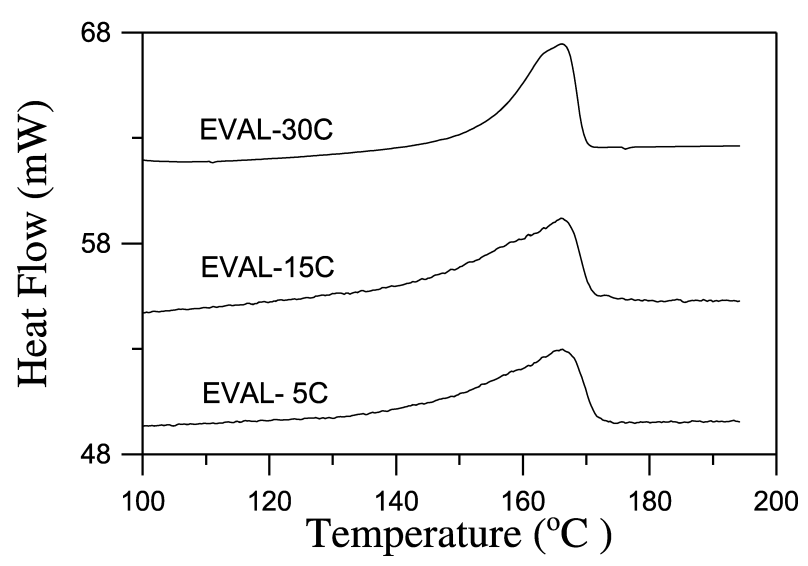

Fig. 6. DSC diagrams of the melting curves of the solid EVAL samples prepared in a closed system at various temperatures. (Scanning rate: $\left.10{ }^{\circ} \mathrm{C} / \mathrm{min}\right)$

the one with an open system was used to mimic the real membrane preparation conditions.

Fig. 4 shows the light transmittance along with the time for the polymer solution in a closed cell equilibrated at various temperatures after the polymer solution was prepared at $60{ }^{\circ} \mathrm{C}$. The changing rate of the light transmittance decreased as the solution temperature increased. There was no change in light transmittance for the solutions kept above $40{ }^{\circ} \mathrm{C}$. The light transmittance decreased gradually along with the time at $35^{\circ} \mathrm{C}$, and it dropped sharply at lower temperatures $\left(\leq 20^{\circ} \mathrm{C}\right)$ due to polymer solid precipitation.

Aggregates of spherical particles of EVAL polymer were obtained and selected SEM pictures of those precipitates (EVAL-30C, EVAL-15C, and EVAL-5C) were shown in Fig. 5. The DSC curves of the melting behaviors of the polymer EVAL-30C, EVAL-15C, and EVAL-5C are shown in Fig. 6, and the corresponding melting heats and peak temperatures are also listed in Table 1. The true densities were $1.100 \pm 0.003 \mathrm{~g} / \mathrm{cm}^{3}$ (Fig. 2) and the porosities were about $0.61( \pm 0.08)$ for all of those samples. Based on the above observation, the spherical polymer particles precipitated at different temperatures were basically the same in terms of crystallinity but were different in size. Those precipitates were formed through the same mechanism.

Clearly, the phase behavior observed here was TIPS. A solid-liquid demixing in the casting solution resulted in the drop of light transmittance. In general, the semi-crystalline polymer particulates were formed by a nucleation and growth mechanism. When the polymer was precipitated from the solution at lower temperatures, more nucleates could form and the size of the particulates were smaller (Fig. 5). The nominal size of the spherical particles was 2.2, 1.0, and $0.8 \mu \mathrm{m}$ for EVAL-30C, EVAL-15C, and EVAL-5C, respectively. The abrupt drop in light transmittance at lower temperatures was attributed to the fast nucleation rate of polymer precipitates. The gradual decrease of light

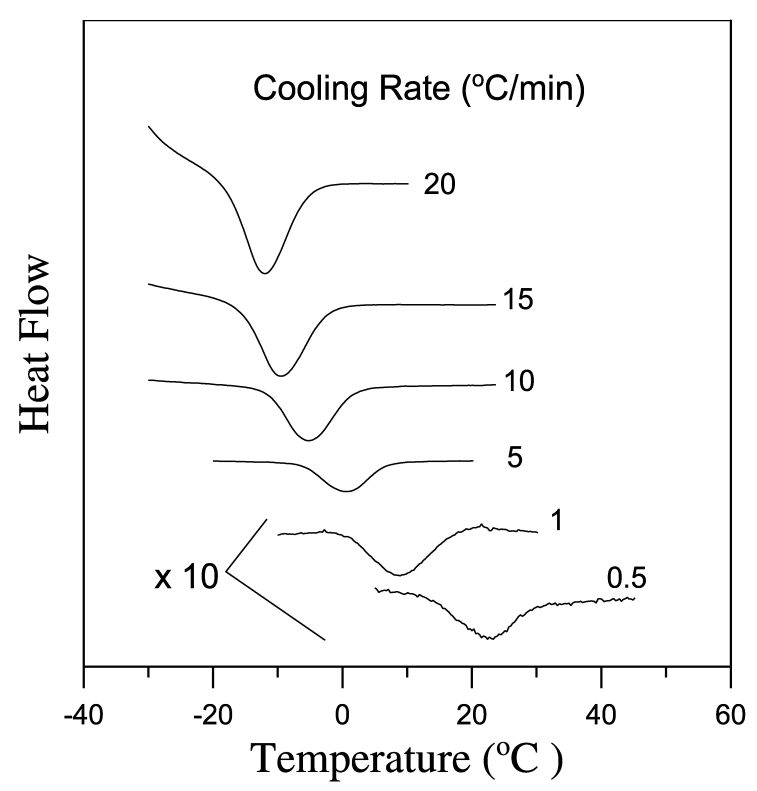

Fig. 7. DSC diagrams of the crystalline curves of the EVAL solutions at various cooling rates.

transmittance at higher temperatures was controlled by the growth rate since less number of nucleates were formed.

The solid polymer precipitated was highly crystalline so that the phase separation was an exothermic process and the phase behavior could be followed by a dynamic DSC study. The thermal behavior of the crystallization depended on the cooling rate of the DSC scan (Fig. 7). The incipient point of the exothermic process was recorded as the dynamic crystallization temperature $\left(T_{\mathrm{c}}\right)$. The observed dynamic crystallization temperatures decreased as the cooling rate increased (Fig. 8). Super-cooling would result in the lower value of the measured crystallization temperature. The equilibrium crystallization temperature could be approximated at infinitely slow cooling rate and it was estimated to be around $35.5^{\circ} \mathrm{C}$.

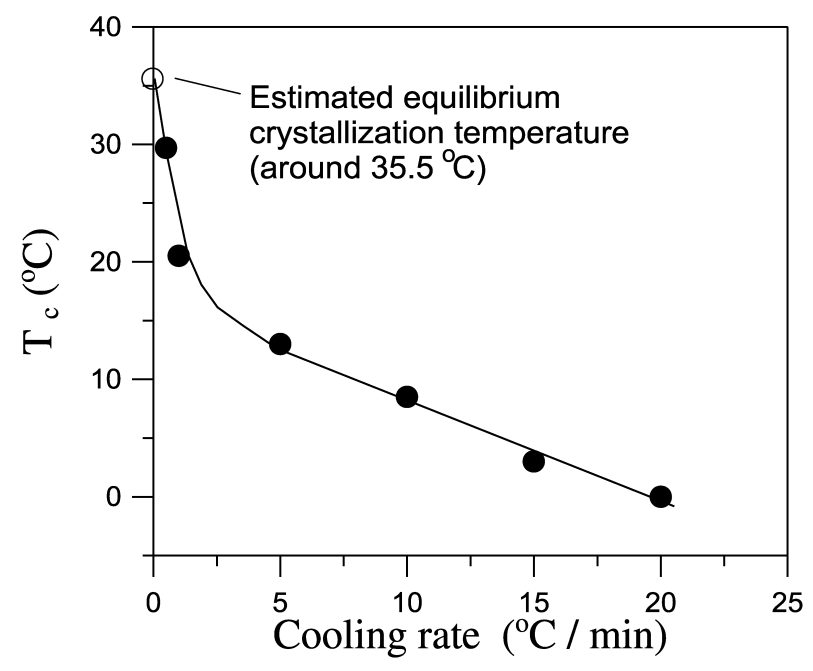

Fig. 8. Dynamic crystallization temperature $\left(T_{\mathrm{c}}\right)$ as a function of cooling rate for EVAL solutions. 


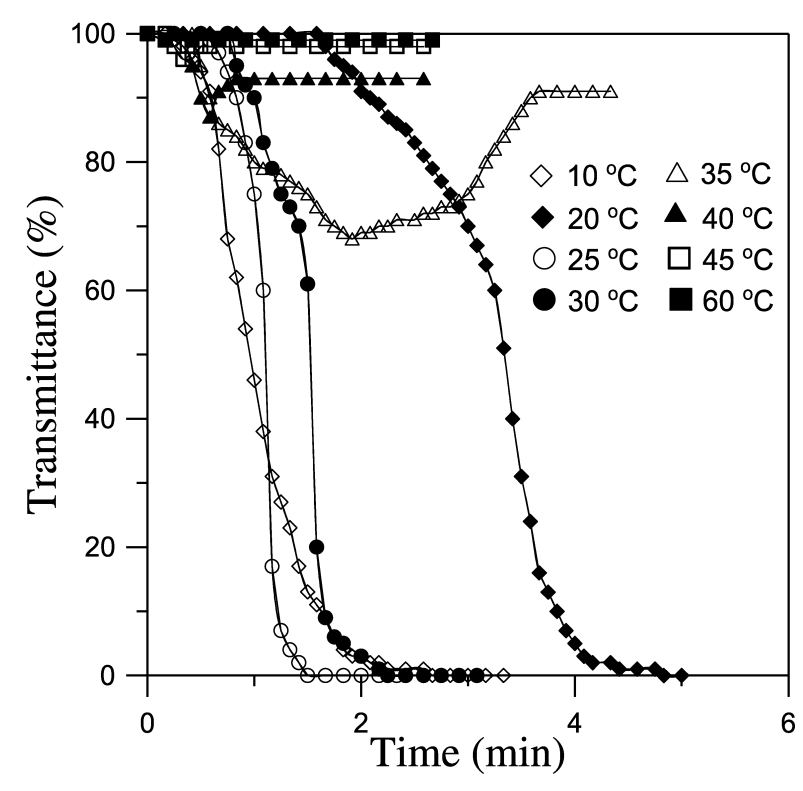

Fig. 9. Light transmittance-time curves for EVAL solutions in an open system at various temperatures.

Fig. 9 illustrates the light transmittance-time curves in the open system study at various temperatures. The phase separation in an open system completed much faster than that in a closed system. The phase separation completed within $4 \mathrm{~min}$ at temperatures of or below $30^{\circ} \mathrm{C}$. In contrast, it took more than $300 \mathrm{~min}$ for the phase separation to complete in a closed system at $30{ }^{\circ} \mathrm{C}$ (Fig. 4). The effect of solvent evaporation from the casting solution is obvious in the membrane preparation process.

It is difficult to completely interpret all the light transmittance-time curves shown in Fig. 9 due to rather limited information about the fast change of phases in a short time. With a comparison of the SEM pictures from those membranes (Fig. 1) with those from polymer block samples (Fig. 5), a tentative explanation of the process of membrane formation is given as follows. At temperatures of 45 or $60{ }^{\circ} \mathrm{C}$, the casting solution was dried without any phase separation and dense membranes were formed when all the solvent was completely evaporated. At temperatures of 35 and $40{ }^{\circ} \mathrm{C}$, the casting solution phase separated immediately after cast, but it might regain its clarity later. The process might involve a path passing through the twophase region in the phase diagram. The solvent in the polymer-lean phase might be evaporated first and the polymer-rich phase collapsed before the polymer was gelled or solidified. At 25 and $30{ }^{\circ} \mathrm{C}$, the casting solution became turbid completely within $2 \mathrm{~min}$. This process can be attributed to a sequence or combination of nucleation and growth and spinodal decomposition liquid-liquid phase separations upon temperature reduction and solvent evaporation. It was unlikely to be solid-liquid demixing because it was still above the dynamic crystallization temperature (Fig. 8) upon such a quick temperature change. The time available for the orientation of the polymer molecules might

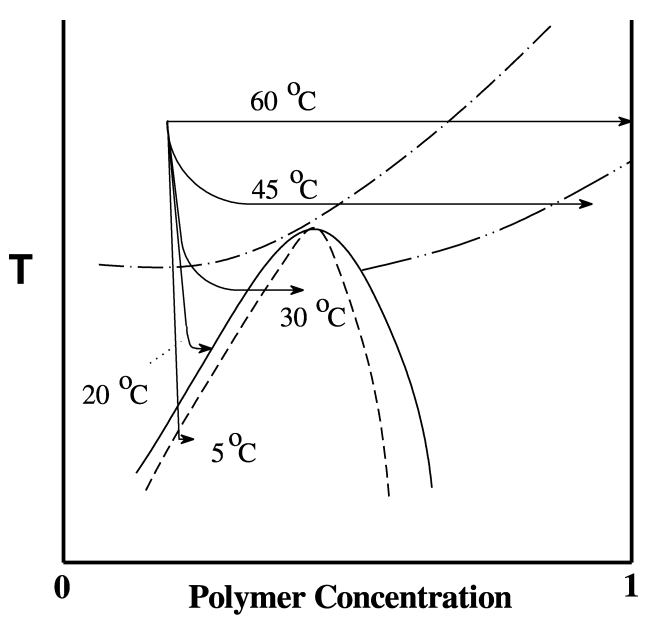

Fig. 10. Schematic phase diagram and membrane formation path for EVAL casting solution. (crystallization boundary: $-\cdot \cdot-\cdot-\cdot ;$ vitrification line: $\cdot \cdot-\cdot \cdot-$; binodal curve: - ; spinodal curve: - - - -).

be too short for the polymer solution to well crystallize and the crystallinity of EVAL-30 was the lowest among all. At $20{ }^{\circ} \mathrm{C}$, the phase separation delayed for another $1 \mathrm{~min}$ and the initial changing rate (between 2 and $3 \mathrm{~min}$ ) of light transmittance was moderate in comparison with those at other temperatures. This process might be due to the slower crystal nucleation and growth since most of the solvent was still retained in the solution during this time period. The structure of the membrane EVAL-20 is the same as that of the polymer samples obtained in a closed system operation (Figs. 1(c) and 5). The light transmittance change should be caused by the solid-liquid demixing, and the process was similar to that in a close system operation. The light transmittance dropped very fast after $3 \mathrm{~min}$ of the experiment when the process reached a point that phase separation could occur instantaneously. The point may correspond to the binodal boundary of the liquid-liquid two-phase region in a phase diagram at $20{ }^{\circ} \mathrm{C}$. At temperatures of $10^{\circ} \mathrm{C}$ or below, the light transmittance dropped instantaneously within $1.3 \mathrm{~min}$. The membrane formed at $5{ }^{\circ} \mathrm{C}(\mathrm{EVAL}-5)$ showed a leaf structure (Fig. 1(d)) but its morphology was highly crystalline as indicated by relatively large melting heat (Table 1 ). The phase separation might be a liquid-liquid demixing and then followed with immediately polymer crystallization.

\subsection{Phase diagram and membrane formation mechanism}

A schematic phase diagram (Fig. 10) of the casting solution is constructed assuming the solution is a binary polymer-solvent system. Although the suggested phase diagram is not an exact one due to limited information, it gives us a hint on the mechanism during membrane formation.

The crystalline EVAL polymer melts at around $165^{\circ} \mathrm{C}$, the equilibrium crystallization temperature of the polymer precipitated from the $15 \%$ polymer solution is about $35^{\circ} \mathrm{C}$, 
and the glass transition temperature of the amorphous EVAL is $55^{\circ} \mathrm{C}$ [21]. The glass transition temperature of pure EVAL may lead a vitrification line for the amorphous portion in the concentrated polymer solution system. When the polymer solution was cast at 60 or $45^{\circ} \mathrm{C}$, the membrane was formed solely by solvent evaporation. The polymer solution became gel when the composition path passed through the crystallization line. A dense membrane was obtained when the solvent completely evaporated. The membrane prepared at $60{ }^{\circ} \mathrm{C}$ (EVAL-60) was formed above the vitrification line so that its polymer chains had chance to reorient in a regular pattern and crystallize. The true density and bulk density of the membrane were the same, which indicated that the membrane was a truly dense membrane. On the other hand, the membrane prepared at $45^{\circ} \mathrm{C}(\mathrm{EVAL}-$ 45) would go across the vitrification line; therefore, amorphous portion of the polymer chains within the membrane might be frozen and loosely packed before all the solvent was removed. Consequently, the crystallinity of EVAL-45 was less than that of EVAL-60. Although the membrane is still transparent and no pore was observed in SEM pictures, it is not a truly dense membrane since a difference was found between its bulk density and true density. It is speculated that micropores of molecular size, which were originally occupied by solvent molecules upon vitrification, were left in the membrane. Those micropores were big enough to accommodate helium, but too small to be observed in SEM.

According to the observations from the light transmittance and SEM experiments, it is most possible that there is a liquid-liquid phase separation region with an UCST slightly above $40{ }^{\circ} \mathrm{C}$. The end of the vitrification line should be below $35^{\circ} \mathrm{C}$ when the line touched the boundary of the two-phase region so that the casting solution at 35 or $40^{\circ} \mathrm{C}$ may regain some of its clarity before solidification (Fig. 9).

For the membrane formed at $30{ }^{\circ} \mathrm{C}$ (EVAL-30), the temperature dropped directly so that the composition path could rapidly pass through the crystallization line. However, solvent evaporation would also bring the solution into liquid-liquid phase separation region. Membrane formation processes are determined by considerations of the thermodynamic (phase behavior) and kinetic (mass transfer) aspects [7]. Under ordinary conditions, crystallization is slower than liquid-liquid demixing because of the time required for orientation of the polymer molecules. In general, initiation of liquid-liquid demixing is more rapid than nucleation of polymer crystallization that requires a rearrangement of polymer [22], so liquid-liquid demixing is kinetically favored to dominate the membrane structure. The polymer-rich phase solution formed the wall of the pores and polymer-lean phase solution became pores after the solvent was completely removed. The temperature should be lower than the vitrification line so that the porous structure could be retained. Although the morphology of EVAL-30 membrane was still semi-crystalline, the corresponding crystallinity (melting heat) was the lowest among all the EVAL membranes or polymer samples obtained in this study. The membrane structure was vitrified along with liquid-liquid phase separation and the crystallization was partially retarded. A combined effect of TIPS and solvent evaporation accounted for the membrane formation process.

For the membranes formed at 20 and $5{ }^{\circ} \mathrm{C}$ (EVAL-20 and EVAL-5), their internal structures were completely different from those formed at higher temperatures. The melting heats of EVAL-20 and EVAL-5 membranes were similar and were close to that obtained of a sample prepared in the closed system. The particulate morphology of EVAL-20 was almost the same as that obtained in a closed system, which indicated that the membrane formation was due to a slower solid-liquid demixing mechanism. From the light transmittance data, a delayed phase separation was observed (Fig. 9). It seemed that the evaporation rate was not fast enough to bring the system into the liquid-liquid demixing region during membrane formation. The dynamic crystallization temperature might approach $20^{\circ} \mathrm{C}$ when the cooling rate was slowed down at the later stage in this case. The solid-liquid demixing occurred before the composition path entered the binodal boundary of the liquid-liquid two-phase region and resulted in a particulate structure. For a membrane formed at $5{ }^{\circ} \mathrm{C}$, the instantaneous phase separation and leafy membrane structure suggested that the casting solution directly fell into the liquidliquid phase separation region and then immediately induced the polymer crystallization. It must be pointed out that the polymer solution was supper-cooled but still above the dynamic crystallization temperature and any fluctuation in polymer concentration could result in crystallization. This implies that the membrane formation is not dictated by liquidliquid demixing only. Solid-liquid demixing due to crystallization has become a competitive membrane formation mechanism. Therefore, liquid-liquid demixing and crystallization occurred nearly simultaneously. In such a case, the formed morphology exhibits features from both types of phase transformations. The leafy or flake-like structure within the membrane was a connected tissue of highly crystalline particulates. Liquid-liquid separation was possible in EVAL-5 membrane formation but not in EVAL-5C because the polymer concentration in the casting solution of EVAL-5 had been slightly concentrated due to solvent evaporation.

With the aid of the proposed phase diagram, we can better interpret the processes of membrane formation at several different temperatures. Solvent evaporation and phase separation are two primary factors determining the resulting membrane structure. Slight change in membrane preparation conditions can lead to significant variation in membrane structure.

\section{Conclusions}

This paper has presented a case of EVAL membrane preparation from a casting solution of EVAL-isopropanolwater system. Depending on the casting temperature, 
membranes of different bulk or true densities, porosity, flexibility, crystallinity, and structure were obtained. TIPS and solvent evaporation were involved in the membrane formation mechanism. The membrane could be truly dense if it was prepared above the glass transition temperature $\left(T_{\mathrm{g}}\right)$ of pure polymer via solvent evaporation (EVAL-60). It might contain micropores if it was prepared, also via solvent evaporation, at a temperature lower than the $T_{\mathrm{g}}$ of pure polymer but higher than the UCST of the liquid-liquid phase separation region (EVAL-45). Due to the concentration change created by a fast solvent evaporation, the casting solution might enter the liquid-liquid demixing region and result in a porous and a least crystalline membrane (EVAL-30). In this case, the membrane preparation temperature is below the UCST but is higher than the dynamic crystallization temperature. The membrane was formed by a combined effect of TIPS with solvent evaporation. As the membrane casting temperature was lowered, it might approach to the dynamic crystallization temperature of the polymer solution. Thermally induced solid-liquid phase separation would result in a highly porous and crystalline membrane of particulate structure (EVAL-20). Thermally induced liquid-liquid phase separation followed by immediately crystallization created a highly porous and crystalline membrane of leafy structure (EVAL-5). The structure of the EVAL membranes could be varied by carefully selecting the membrane casting temperature; therefore, the properties of the membranes could be manipulated for specific applications.

This study also demonstrated a case that microporous polymer membranes can be fabricated simply by the solution casting method in combination of TIPS with solvent evaporation. The method can be applied to a polymer-solvent system with a phase separation region in the phase diagram. A relatively volatile solvent is used so that it can be removed during membrane formation. Depending on the casting temperature, the structure and morphology of the membranes can be adjusted. Comparing to the immersion precipitation method, the use of a nonsolvent is avoided. In comparison with the conventional TIPS method, an afterward solvent-extraction procedure to remove the nonvolatile diluents is avoided. The proposed membrane preparation method is relatively simple in operation and is worth further studying in preparation of other microporous polymer membranes.

\section{Acknowledgements}

The authors gratefully acknowledge the financial support provided by the China Petroleum Cooperation and National Science Council of the Republic of China through the grant of NSC-89-CPC-7-155-002.

\section{References}

[1] Kesting RE. Synthetic polymer membranes. New York: Wiley; 1985.

[2] Mulder M. Basic principles of membrane technology. Dordrecht: Kluwer; 1991.

[3] Baker RW. Membrane technology and applications. New York: McGraw-Hill; 2000.

[4] Leob S, Sourirajan S. U.S. Patent 3,133,132, 1964

[5] Strathmann H, Scheible P, Baker RW. J Appl Polym Sci 1971;15:811.

[6] Altena FW, Smolders CA. Macromolecules 1982;15:1491.

[7] Reuvers AJ, van der Berg JWA, Smolders CA. J Membr Sci 1987;34: 45.

[8] Caneba GT, Soong DS. Macromolecules 1985;18:2538.

[9] Tsai FJ, Torkelson JM. Macromolecules 1990;23:775.

[10] Lloyd DR, Kinzer KE, Tseng HS. J Membr Sci 1990;52:239.

[11] Lloyd DR, Kim SS, Kinzer KE. J Membr Sci 1991;64:1.

[12] Matsuyama H, Teramoto M, Kudari S, Kitamura Y. J Appl Polym Sci 2001;82:169.

[13] Young TH, Lai JY, Yu WM, Cheng LP. J Membr Sci 1997;128:55.

[14] Young TH, Cheng LP, You WM, Chen LY. Polymer 1999;40:2189.

[15] Young TH, Lin DT, Chen LY, Huang YH, Chiu WY. Polymer 1999; 40:5257.

[16] Young TH, Huang YH, Chen LY. J Membr Sci 2000;164:111.

[17] Young TH, Huang YH, Huang YS. J Membr Sci 2000;171:197.

[18] Cheng LP, Young TH, Chung WY, Chen LY, Chen LW. Polymer 2001;42:443.

[19] Young TH, Cheng LP, Hsieh CC, Chen LW. Macromolecules 1998; 31:1229.

[20] Young TH, Hsieh CC, Chen LY, Huang YS. J Membr Sci 1999;159: 21.

[21] Luo RL. MS Thesis, Yuan Ze University, Chung-Li, 2001.

[22] Reuvers AJ, Altena FW, Smolders CA. J Polym Sci Polym Phys 1986; 24:793. 